\title{
Best reproducibility of the ankle-arm index was calculated using Doppler and dividing highest ankle pressure by highest arm pressure
}

\author{
Femke Atsma, Marie-Louise E.L. Bartelink*, Diederick E. Grobbee, Yvonne T. van der Schouw \\ Julius Center for Health Sciences and Primary Care, University Medical Center Utrecht, P.O. Box 85500, Str. 6.131, 3508 GA Utrecht, The Netherlands \\ Accepted 4 April 2005
}

\begin{abstract}
Background and Objective: To investigate which method of ankle-arm index (AAI) measurement in terms of selected arteries and blood pressure devices yields the highest reproducibility.

Study Design and Setting: In this cross-sectional study, duplicate AAI measurements were obtained at the right and left side in 320 postmenopausal women. Analyses were done as proposed by Bland and Altman and intraclass correlation coefficients were calculated.

Results: The mean and standard deviation (SD) of the first and second systolic blood pressure (SBP) measurement separately did not differ from the mean and SD of the duplicate SBP measurements averaged. Coefficients of repeatability and intraclass correlation coefficients were comparable when the ankle SBP was measured in the posterior tibial artery or dorsalis pedis artery. Coefficients of repeatability as well as the intraclass correlation were better when the SBP in the brachial artery was measured with Doppler than with Dinamap.

Conclusions: To achieve the best reproducibility and smallest systematic difference in the AAI, single SBP measurements should be obtained by Doppler in the brachial artery, posterior tibial artery and dorsalis pedis artery. The AAI should be calculated for each foot separately by dividing the highest ankle SBP by the highest arm SBP. (c) 2005 Elsevier Inc. All rights reserved.
\end{abstract}

Keywords: Blood pressure; Ankle-arm index; Reproducibility; Peripheral vascular diseases; Women

\section{Introduction}

The measurement of the ankle-arm index (AAI), a simple and inexpensive test, is increasingly used as an objective measure for the diagnosis of peripheral arterial disease [1-5]. Furthermore, a low AAI independently predicts cardiovascular morbidity and mortality and total mortality [6-9]. In spite of its importance, the measurement of AAI is not well standardized and various methods of AAI measurements have been described in the literature. Inconsistency exists about the selection of arteries, the blood pressure device to be used, and the value of duplicate measurements. The ankle systolic blood pressure (SBP) can be obtained at the posterior tibial artery or at the dorsalis pedis artery in both feet using a handheld 8-MHz Doppler probe. The SBP at the arm can be measured at the right and left brachial artery, and can be measured auscultatorily, or

* Corresponding author. Tel.: +31-30-2538383; fax: +31-302539028.

E-mail address: M.E.L.Bartelink@umcutrecht.nl (M.E.L. Bartelink). using a handheld 8-MHz Doppler probe, or using a device such as a Dinamap. Due to the variability in methods for AAI measurements, the mode of calculation is not well established either.

Reproducibility is one of the most important qualities of a diagnostic and prognostic test. In the present study, we investigated which method of AAI measurement, in terms of selected arteries and device, yields the lowest intraobserver variability and thus the best reproducibility and whether duplicate measurements may increase reproducibility.

\section{Methods}

\subsection{Design and participants}

Participants were recruited from the PROSPECT study, one of the two Dutch cohorts participating in the European Prospective Investigation into Cancer and nutrition (EPIC) [10]. In PROSPECT, a total of 17,357 healthy breast-cancer screening participants, aged 49-70 years, living in Utrecht and surroundings, were enrolled between 1993 and 1997. The purpose of the PROSPECT-EPIC study is to assess the relation between nutrition and cancer and other chronic diseases. 


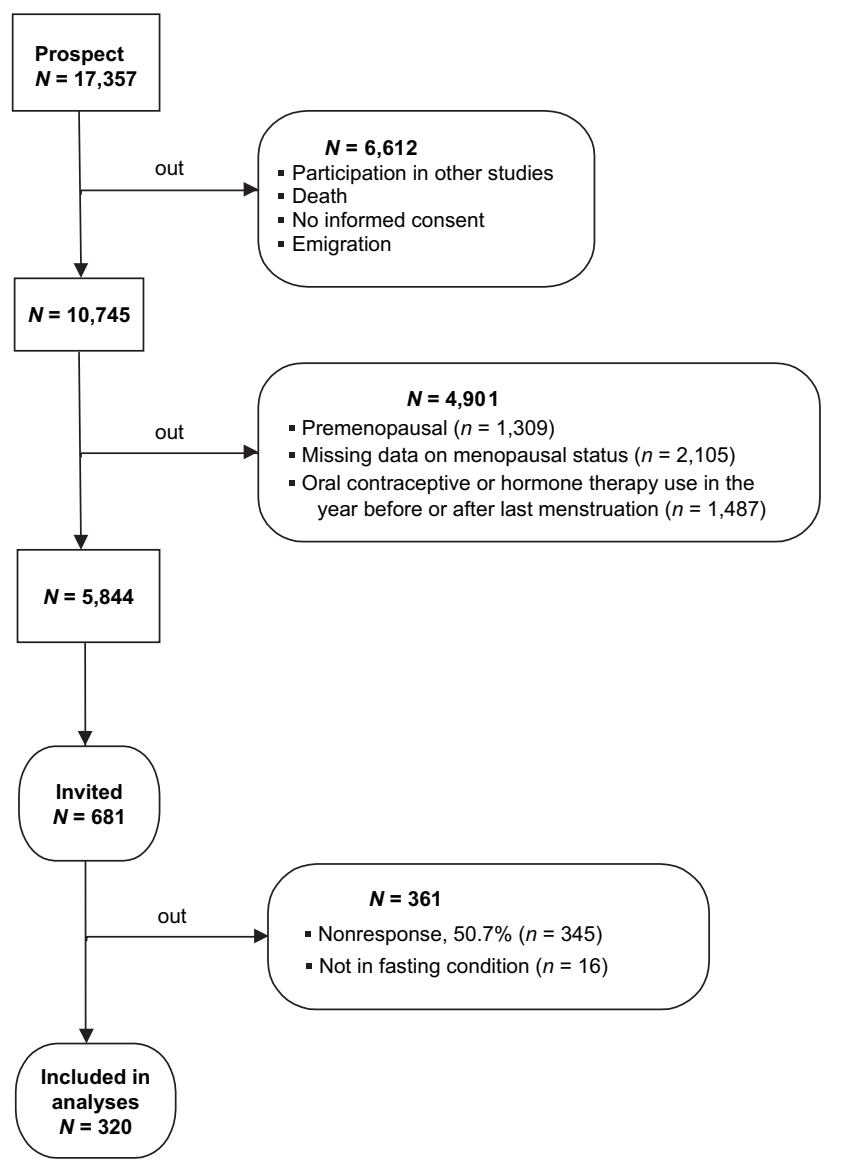

Fig. 1. Flowchart for selection of the study population.

In Fig. 1, the selection procedure of our study population is outlined. Of the 17,357 women 6,612 women were excluded because of death, participation in other studies, absence of written informed consent, or emigration. Other reasons for exclusion were premenopausal state $(n=$ $1,309)$, missing data on menopausal status $(n=2,105)$ or use of oral contraceptives (OC) or postmenopausal hormone therapy $(\mathrm{HT})$ in the year before or after the last menstruation ( $n=1,487)$. Out of 5,844 eligible women, a random selection of 681 women were invited for the present study by a personal letter from the principal investigator of PROSPECT and 336 (49,3\%) answered positively. Participants who were not in fasting condition $(n=16)$ were excluded. The final study population comprised 320 women.

The study was approved by the Institutional Review Board of the University Medical Center Utrecht and written informed consent was obtained from all participants. Data collection took place between October 2002 and March 2003.

Hypertension was defined as a systolic blood pressure $>$ $160 \mathrm{mmHg}$ and/or diastolic blood pressure $>95 \mathrm{mmHg}$ and/or use of antihypertensive drugs. Diabetes mellitus was defined as a fasting blood glucose $>6.9 \mathrm{mmol} / \mathrm{L}$ and/or use of antidiabetic medication.

\subsection{AAI measurement}

To investigate intraobserver variability, in all subjects duplicate AAI measurements were performed by one trained examiner during one visit. In total, three examiners were involved in the performance of all AAI measurements.

Each participant had her AAI measured in fasting condition and after lying supine for 10 minutes. Fasting was defined as not eating, drinking, or smoking within the 8 hours before examination. The ankle SBP was measured using an 11-cm-wide blood pressure cuff and were measured manually in the right and left posterior tibial artery and in the right and left dorsalis pedis artery using a handheld 8-MHz Doppler probe (Imexdop CT+, Nicolet Vascular, Golden, CO, USA) and an aneroid sphygmomanometer (Welch Allyn, Skaneateles, NY, USA). Brachial blood pressure measurements were performed in both arms according to two methods: automatically, using a Dinamap device (Critikon Dinamap XL; Johnson \& Johnson, Tampa, FL, USA), and manually, using a handheld 8-MHz Doppler probe and the aneroid sphygmomanometer.

All measurements were performed twice in the following order: left arm (Dinamap), right arm (Doppler), right foot (dorsalis pedis artery), right foot (posterior tibial artery), right arm (Dinamap), left arm (Doppler), left foot (dorsalis pedis artery), left foot (posterior tibial artery).

\subsection{Statistical analysis}

To investigate whether duplicate measurements reduce variability over single measurements, mean and standard deviation (SD) of the two single measurements separately were compared with the mean and SD of the duplicate measurements averaged.

Reproducibility of duplicate SBP measurements was assessed at eight locations: posterior tibial artery (right and left), dorsalis pedis artery (right and left), and brachial artery (right and left) measured with Dinamap and the brachial artery (right and left) measured with Doppler. Agreement between Dinamap and Doppler in each arm when measuring brachial SBP was also assessed, and the reproducibility of the AAI as a whole, instead of the reproducibility of its different components, was determined. Ten methods of AAI measurement and AAI calculation were investigated, right and left. This includes the comparison between the brachial SBP taken by Doppler and Dinamap in the denominator and the comparison between the tibial posterior artery and the dorsalis pedis artery in the numerator. For the latter, we compared single, highest, lowest, and average SBP values of these two arteries in the numerator of the AAI. Finally, we assessed the intraobserver variability of the AAI measurement for the three examiners separately. We compared the three examiners on the modes of AAI calculation that performed best in the overall analyses. 
Repeatability of duplicate measurements and the agreement between Dinamap and Doppler for measuring the brachial SBP were analyzed according to the methods proposed by Bland and Altman [11]. First, the distribution of differences between two measurements was assessed by drawing histograms. In case of a skewed distribution, the data were $\log$ transformed. To examine repeatability of duplicate measurements, coefficients of repeatability (CR) were calculated. When investigating repeatability, the mean difference between the duplicate measurements should not be significantly different from zero. Furthermore, a plot of the difference between Doppler and Dinamap against their mean was constructed, in which the mean difference and the limits of agreement are shown. To investigate whether there are systematic differences between Doppler and Dinamap, mean differences and $95 \%$ confidence intervals (85\% CI) were obtained by one-sample $t$-tests [11]. Single SBP measurements (the first measurement) were used to compare the two methods.

In addition, intraclass correlation coefficients (ICC) and 95\% confidence intervals were calculated. When repeatability of duplicate measurements was investigated intraclass correlation coefficients were calculated according to the "two-way random effect model" and the "absolute agreement definition." When Doppler and Dinamap were compared intraclass correlation coefficients were calculated according to the "two-way mixed effect model" and the "consistency definition."

\section{Results}

\subsection{Population characteristics}

The mean age of the subjects was $65.5( \pm 5.7)$ years. Of the study population $(n=320), 34.4 \%$ had hypertension, $7.2 \%$ had diabetes, and $0.9 \%$ had a history of coronary heart disease. All differences between blood pressure measurements were normally distributed. Table 1 shows that no differences could be observed between the mean and SD of the first and second SBP measurement separately compared to the mean and SD of the of the duplicate SBP measurements averaged.

\subsection{Repeatability of duplicate SBP measurements}

The repeatability of duplicate SBP measurements in the right and left posterior tibial artery and in the right and left dorsalis pedis artery is shown in Table 2 . In all four foot arteries, mean differences between duplicate SBP measurements did not differ significantly from zero. CRs were $11.1 \mathrm{mmHg}$ and $11.2 \mathrm{mmHg}$ for the right and left posterior tibial artery and $14.0 \mathrm{mmHg}$ and $14.4 \mathrm{mmHg}$ for the right and left dorsalis pedis artery, respectively. There were no differences in intraclass correlation coefficients (ICC) on the agreement of duplicate measurements between tibial posterior artery and dorsalis pedis artery for both right and left side.

In Table 3, the reproducibility of duplicate SBP measurements in the brachial artery using Dinamap and the reproducibility of duplicate brachial SBP measurements obtained with Doppler are shown. Mean differences between duplicate SBP measurements in the brachial artery were not significantly different from zero, irrespective of the method used. The CRs of the Dinamap (CR: 18.1 $\mathrm{mmHg}$ right arm and $17.6 \mathrm{mmHg}$ left arm) were considerably larger than the CRs for Doppler (CR: 13.1 $\mathrm{mmHg}$ right arm and $11.8 \mathrm{mmHg}$ left arm). Furthermore, the intraclass correlation coefficients for the agreement between duplicate measurements were slightly better for Doppler (right: $\mathrm{ICC}=0.95,95 \% \mathrm{CI}=0.94-0.96$ ); left: ICC $=0.96,95 \%$ CI $=0.95-0.97$ ) than for Dinamap (right and left: $\mathrm{ICC}=0.92,95 \% \mathrm{CI}=0.90-0.93$ ).

\subsection{Agreement between Dinamap and Doppler in each arm}

Table 4 compares SBP measurements obtained with Dinamap and the SBP obtained with Doppler in the same arm. SBP measured with Dinamap was systematically lower than Doppler when measuring at the same arm. This difference was more pronounced at the right site (mean SBP difference: $-6.0 \mathrm{mmHg}, 95 \% \mathrm{CI}=-7.4$ to 4.6 ). The limits of agreement were also wide $(-31.5$ to $19.6 \mathrm{mmHg})$. At the left side, the mean SBP difference was $-1.7 \mathrm{mmHG}$ (95\% CI $=-2.9$ to -0.6 ), with limits of agreement ranging from -23.0 to $19.5 \mathrm{mmHg}$. The intraclass correlation coefficient (ICC) for the agreement between Dinamap and Doppler in each arm was somewhat higher at the left side ( $\mathrm{ICC}=0.87,95 \% \mathrm{CI}=0.85-0.90)$ than at the right side (ICC $=0.83,95 \% \mathrm{CI}=0.79-0.86$ ).

\subsection{Reproducibility of AAI measurement}

Table 5 presents different methods of AAI measurement and AAI calculation. The results show that for both right and left side the AAI performed better when the SBP in the brachial artery was measured with Doppler as opposed to Dinamap. When we investigated the dorsalis pedis artery and the posterior tibial artery separately in the numerator, there was no difference in performance. After taking the lowest, highest, or average SBP of the two arteries in each leg, the reproducibility increased a little, but did not differ materially between the three methods.

\subsection{Intraobserver variability of three separate examiners}

Table 6 compares the reproducibility of the three examiners separately according to the three best modes of AAI calculation; the highest, lowest, and average SBP of the posterior tibial artery and dorsalis pedis artery divided 
Table 1

Systolic blood pressure in different arteries, measured twice, and with different methods

\begin{tabular}{|c|c|c|c|c|}
\hline \multirow[b]{2}{*}{ Artery and method } & \multirow[b]{2}{*}{$N$} & \multicolumn{3}{|c|}{$\mathrm{SBP}$, mean $\pm \mathrm{SD}, \mathrm{mmHg}$} \\
\hline & & Meas. 1 & Meas. 2 & Meas. 1 and 2, averaged \\
\hline Posterior tibial artery, right & 316 & $152.4 \pm 21.0$ & $152.7 \pm 21.1$ & $152.5 \pm 20.9$ \\
\hline Posterior tibial artery, left & 315 & $152.4 \pm 20.7$ & $152.7 \pm 21.0$ & $152.6 \pm 20.7$ \\
\hline Dorsalis pedis artery, right & 317 & $149.9 \pm 21.7$ & $150.6 \pm 21.5$ & $150.2 \pm 21.3$ \\
\hline Dorsalis pedis artery, left & 315 & $148.9 \pm 22.2$ & $148.3 \pm 22.2$ & $148.6 \pm 22.0$ \\
\hline Brachial artery, Dinamap, right & 316 & $132.9 \pm 21.8$ & $133.6 \pm 22.2$ & $133.3 \pm 21.6$ \\
\hline Brachial artery, Dinamap, left & 316 & $134.8 \pm 21.6$ & $134.6 \pm 21.4$ & $134.7 \pm 21.0$ \\
\hline Brachial artery, Doppler, right & 316 & $139.0 \pm 22.0$ & $139.6 \pm 21.9$ & $139.3 \pm 21.7$ \\
\hline Brachial artery, Doppler, left & 314 & $136.6 \pm 20.9$ & $136.2 \pm 20.8$ & $136.4 \pm 20.6$ \\
\hline
\end{tabular}

The sample size was 320. Reasons for missing blood pressure measurements were amputation, pain due to measurement, absent or weak Doppler signal, varices, and lymph gland resection.

Abbreviations: Meas., measurement; SBP, systolic blood pressure; SD, standard deviation.

by the highest brachial SBP measured with Doppler. There were clear differences between the examiners. Examiner 2 performed best, with coefficients of repeatability varying from 0.05 to 0.07 and intraclass correlation coefficients varying from $0.92(95 \% \mathrm{CI}=0.87-0.96)$ to 0.94 (95\% CI $=0.88-0.97$ ), examiner 3 performed worst with coefficients of repeatability varying from 0.12 to 0.20 and intraclass correlation coefficients varying from 0.60 (95\% $\mathrm{CI}=0.33-0.78)$ to $0.78(95 \% \mathrm{CI}=0.60-0.89)$.

\section{Discussion}

The present study shows that there was no marked difference in mean and SD between a single SBP measurement separately and the mean and SD of duplicate measurements averaged. The reproducibility of duplicate measurements did not differ materially when the ankle SBP was measured in the posterior tibial artery or dorsalis pedis artery. Furthermore, the Doppler method seemed to be more reproducible than the Dinamap when measuring brachial SBP. Moreover, the Dinamap yielded a SBP that was systematically lower than Doppler when measured at the same arm.

To our knowledge, this is the first study in which the reproducibility of all different components of the anklearm index has been evaluated in a same-study population of healthy participants. A maximal effort was made to reduce errors and variability inherent to indirect blood pressure measurements in general. Systematic error may be caused by (i) observer bias, (ii) last digit preference, (iii) problems with equipment, or (iv) variation in used technique $[12,13]$. Random variation reflects fluctuations in biological, mechanical and environmental circumstances, such as seasonal variation, body mass index, use of antihypertensive drug therapy, plasma cholesterol, smoking status, and diabetes [14-16]. In our study, AAI measurements were performed by a well-trained and experienced observer. All equipment was checked and calibrated at fixed intervals and the used equipment was held the same for all participants. Furthermore, we investigated reproducibility within fasting subjects in whom all measurements were performed during a 1-hour visit after lying down for 10 minutes.

Our finding that a single measurement is sufficient compared to duplicate measurements within a short time interval is supported by previous reports, where triplicate and duplicate measurements were not superior over single measurements $[17,18]$. We therefore conclude that a single measurement per visit is suitable in clinical practice and for most epidemiological studies on atherosclerotic peripheral arterial disease.

Based on our results, we did not find a material difference in the reproducibility of SBP measurement between the posterior tibial artery and the dorsalis pedis artery. According to the coefficients of repeatability, measurement with the posterior tibial artery seems to be

Table 2

Repeatability of duplicate SBP measurements in different foot arteries, Doppler method

\begin{tabular}{lllll}
\hline Artery & $N$ & $\begin{array}{l}\text { Mean difference in SBP, } \\
\text { mean }(95 \% \mathrm{CI}), \mathrm{mmHg}\end{array}$ & CR, mmHg & 11.1 \\
\hline Posterior tibial artery, right & 316 & $-0.3(-0.9$ to 0.3$)$ & 11.2 & $0.96(0.95-0.97)$ \\
Posterior tibial artery, left & 315 & $-0.3(-0.9$ to 0.3$)$ & 14.0 & $0.96(0.95-0.97)$ \\
Dorsalis pedis artery, right & 317 & $-0.7(-1.5$ to 0.1$)$ & 14.4 & $0.95(0.93-0.96)$ \\
Dorsalis pedis artery, left & 315 & $0.6(-0.2$ to 1.4$)$ & $0.95(0.93-0.96)$ \\
\hline
\end{tabular}

Abbreviations: CI, confidence interval; CR, coefficient of repeatability; ICC, intraclass correlation coefficient; SBP, systolic blood pressure. 
Table 3

Repeatability of duplicate SBP measurements in the brachial artery, measured with Doppler and Dinamap

\begin{tabular}{|c|c|c|c|c|c|c|c|c|}
\hline \multirow[b]{2}{*}{ Brachial artery } & \multicolumn{4}{|c|}{ Dinamap } & \multicolumn{4}{|c|}{ Doppler } \\
\hline & $N$ & $\begin{array}{l}\text { Mean SBP difference, } \\
\mathrm{mmHg}(95 \% \mathrm{CI})\end{array}$ & $\mathrm{CR}, \mathrm{mmHg}$ & ICC (95\% CI) & $N$ & $\begin{array}{l}\text { Mean SBP difference, } \\
\mathrm{mmHg}(95 \% \mathrm{CI})\end{array}$ & $\mathrm{CR}, \mathrm{mmHg}$ & ICC (95\% CI) \\
\hline Right & 316 & $-0.8(-1.8$ to 0.2$)$ & 18.1 & $0.92(0.90-0.93)$ & 316 & $-0.6(-1.3$ to 0.1$)$ & 13.1 & $0.95(0.94-0.96)$ \\
\hline Left & 316 & $0.2(-0.8$ to 1.2$)$ & 17.6 & $0.92(0.90-0.93)$ & 314 & $0.4(-0.3$ to 1.1$)$ & 11.8 & $0.96(0.95-0.97)$ \\
\hline
\end{tabular}

Abbreviations: CI, confidence interval; CR, coefficient of repeatability; ICC, intraclass correlation coefficient; SBP, systolic blood pressure.

more reproducible than the dorsalis pedis artery; judged by the intraclass correlation coefficient, however, there is no difference in performance. The results did not materially differ when the lowest, highest, or mean SBP from the posterior tibial artery and the dorsalis pedis artery was used as the numerator of the AAI. Two previous studies investigated different modes of AAI calculation in patients with a history of peripheral arterial disease. The results pointed out that the reproducibility of the AAI was best when the mean of SBP in the posterior tibial artery and dorsalis pedis artery in each leg was taken as the numerator for the AAI $[19,20]$. When assessing generalized atherosclerosis, however, in our opinion the aim is to detect an occlusion in the major arteries of the leg and not isolated in the posterior tibial artery or the dorsalis pedis artery. We therefore plead that the higher measurement of the two ankle arteries should be taken instead of the mean or lower of the two ankle arteries. This view is also supported by others [21,22]. In a study of Aboyans et al. [21], the best way of AAI calculation in the general population was investigated, rather than in patients with a history of peripheral arterial disease; the authors recommended taking the highest of the two ankle arteries as the numerator of the AAI.

In our study, measuring with Doppler yielded smaller coefficients of repeatability and larger intraclass coefficients than measuring with Dinamap. These results suggest that Doppler is more reproducible than the Dinamap. Our assistant was not blinded and therefore repeated measurements when using the more subjective Doppler method might not have been independent. In our opinion, however, it is not likely that this affected the results of our study, because the mean differences and their $95 \%$ confidence intervals for duplicate measurements were almost the same for Dinamap and Doppler.

In case of no material difference in brachial SBP between Dinamap and Doppler, the Dinamap would be preferred because it is quick and convenient in clinical practice; however, our results show that Dinamap produces a systematically lower SBP than Doppler. Furthermore, the reproducibility of the AAI reduces when Dinamap was used to measure brachial SBP. Doppler is the method used at the ankle, and therefore Doppler cannot be replaced by Dinamap at the arm, because this would lead to an overestimation of the AAI. This conclusion concurs with a study by Jeelani et al. [23] investigating the usefulness of the Dinamap for brachial SBP measurements for AAI purposes. The results pointed to both an underestimation of brachial SBP and an overestimation of the AAI when Dinamap was used for brachial SBP measurement compared to Korotkoff and Doppler methods.

We found clear differences in intraobserver variability between the three examiners. Examiner 3 had the highest intraobserver variability and examiner 2 the lowest. Although all examiners were trained for the AAI measurement, examiner 3 was the least experienced research nurse and examiner 2 was the oldest and most experienced research nurse. From literature, it is known that lack of experience may negatively influence the reproducibility of the AAI measurement [24,25].

Finally, an interarm difference is commonly reported through literature, with consistently higher SBP values for the right arm than for the left [26-28]. A plausible reason for the right-left differences might be that most people are

Table 4

Agreement between two measurement methods, Dinamap and Doppler

\begin{tabular}{lllll}
\hline Brachial artery & $N$ & $\begin{array}{l}\text { Mean difference in SBP, } \\
\operatorname{mmHg}(95 \% \text { CI })\end{array}$ & $\begin{array}{l}\text { Limits of agreement, } \\
\mathrm{mmHg}\end{array}$ & ICC (95\% CI) \\
\hline Right & 317 & $-6.0(-7.4$ to -4.6$)$ & $-31.5-19.6$ & $0.83(0.79-0.86)$ \\
Left & 314 & $-1.7(-2.9$ to -0.6$)$ & $-23.0-19.5$ & $0.87(0.85-0.90)$ \\
\hline
\end{tabular}

Abbreviations: CI, confidence interval; ICC, intraclass correlation coefficient.

${ }^{a}$ For each side, differences between the Dinamap and Doppler are calculated by subtracting the first brachial SBP obtained by Doppler from the first brachial SBP obtained by Dinamap. 
Table 5

Repeatability of duplicate AAI measurements

\begin{tabular}{llll}
\hline & & Repeatability & \\
\cline { 2 - 4 } Mode of AAI calculation & $N$ & CR & 0.12 \\
PTA/BA Doppler right & 316 & 0.12 & $0.82(0.78-0.85)$ \\
PTA/BA Doppler left & 315 & 0.13 & $0.81(0.77-0.85)$ \\
DPA/BA Doppler right & 317 & 0.13 & $0.83(0.79-0.86)$ \\
DPA/BA Doppler left & 315 & 0.15 & $0.83(0.79-0.86)$ \\
PTA/BA Dinamap right & 316 & 0.15 & $0.75(0.70-0.80)$ \\
PTA/BA Dinamap left & 315 & 0.15 & $0.75(0.70-0.80)$ \\
DPA/BA Dinamap right & 316 & 0.16 & $0.76(0.71-0.81)$ \\
DPA/BA Dinamap left & 315 & 0.12 & $0.75(0.69-0.79)$ \\
Lowest of PTA and DPA/BA Doppler right & 317 & 0.12 & $0.82(0.78-0.85)$ \\
Lowest of PTA and DPA/BA Doppler left & 315 & 0.11 & $0.83(0.79-0.86)$ \\
Highest of PTA and DPA/BA Doppler right* & 317 & 0.12 & $0.84(0.81-0.87)$ \\
Highest of PTA and DPA/BA Doppler left & 315 & 0.10 & $0.82(0.77-0.85)$ \\
Mean of PTA and DPA/BA Doppler right & 316 & 0.10 & $0.85(0.82-0.88)$ \\
Mean of PTA and DPA/BA Doppler left & 315 & 0.15 & $0.84(0.81-0.87)$ \\
Lowest of PTA and DPA/BA Dinamap right & 317 & 0.15 & $0.77(0.72-0.81)$ \\
Lowest PTA and DPA/BA Dinamap left & 315 & 0.14 & $0.76(0.71-0.81)$ \\
Highest of PTA and DPA/BA Dinamap right & 317 & 0.14 & $0.75(0.69-0.79)$ \\
Highest of PTA and DPA/BA Dinamap left & 315 & 0.14 & $0.72(0.67-0.77)$ \\
Mean of PTA and DPA/BA Dinamap right & 316 & 0.14 & $0.76(0.73-0.82)$ \\
Mean of PTA and DPA/BA Dinamap left & 315 & $0.76(0.71-0.80)$ \\
\hline
\end{tabular}

Abbreviations: BA, brachial artery; CI, confidence interval; CR, coefficient of repeatability; DPA, dorsalis pedis artery ICC, intraclass correlation coefficient; PTA, posterior tibial artery.

right handed and, therefore, have a larger muscle mass in the right arm that is less easily compressed by the blood pressure cuff [28]. In individual cases, there are rare anatomical abnormalities and conditions which may lead to a disparity in blood pressure between the two arms, including aortic dissection, congenital heart disease, peripheral vascular disease, and unilateral neurological and musculoskeletal abnormalities [29]. Because subclavian stenosis can be suspected in the arm with lower SBP, it has been recommended that the initial blood pressure should be taken in both arms and that the highest value should be used to guide further decisions [22,28-30].

In conclusion, the results of our study lead to the following recommendations for AAI measurement: (i) single SBP measurements should be obtained by Doppler in the right and left arm and right and left feet; (ii) the ankle SBP should be measured in the posterior tibial artery and in the dorsalis pedis artery; and (iii) the AAI should be calculated for each foot separately, dividing the highest ankle SBP by the highest brachial SBP.

Table 6

Repeatability of duplicate AAI measurements according to three observers

\begin{tabular}{|c|c|c|c|c|c|c|c|c|c|}
\hline \multirow[b]{3}{*}{ Mode of AAI calculation } & \multicolumn{9}{|c|}{ Repeatability } \\
\hline & \multicolumn{3}{|c|}{ Examiner 1} & \multicolumn{3}{|c|}{ Examiner 2} & \multicolumn{3}{|c|}{ Examiner 3} \\
\hline & $N$ & $\mathrm{CR}$ & ICC (95\% CI) & $N$ & $\mathrm{CR}$ & ICC $(95 \%$ CI $)$ & $N$ & $\mathrm{CR}$ & $\operatorname{ICC}(95 \% \mathrm{CI})$ \\
\hline $\begin{array}{l}\text { Lowest of PTA and DPA/BA } \\
\text { Doppler right }\end{array}$ & 231 & 0.12 & $0.84(0.79-0.87)$ & 50 & 0.06 & $0.93(0.88-0.96)$ & 33 & 0.20 & $0.60(0.33-0.78)$ \\
\hline $\begin{array}{l}\text { Lowest of PTA and DPA/BA } \\
\text { Doppler left }\end{array}$ & 230 & 0.12 & $0.78(0.72-0.83)$ & 49 & 0.07 & $0.92(0.87-0.96)$ & 33 & 0.12 & $0.78(0.60-0.89)$ \\
\hline $\begin{array}{l}\text { Highest of PTA and DPA/BA } \\
\text { Doppler right }\end{array}$ & 231 & 0.12 & $0.82(0.77-0.83)$ & 50 & 0.05 & $0.94(0.88-0.97)$ & 33 & 0.13 & $0.78(0.60-0.89)$ \\
\hline $\begin{array}{l}\text { Highest of PTA and DPA/BA } \\
\text { Doppler left }\end{array}$ & 230 & 0.11 & $0.80(0.75-0.84)$ & 49 & 0.07 & $0.92(0.87-0.96)$ & 33 & 0.16 & $0.62(0.35-0.79)$ \\
\hline $\begin{array}{l}\text { Mean of PTA and DPA/BA } \\
\text { Doppler right }\end{array}$ & 230 & 0.11 & $0.85(0.81-0.88)$ & 50 & 0.05 & $0.94(0.89-0.97)$ & 33 & 0.14 & $0.73(0.52-0.86)$ \\
\hline $\begin{array}{l}\text { Mean of PTA and DPA/BA } \\
\text { Doppler left }\end{array}$ & 230 & 0.11 & $0.81(0.76-0.85)$ & 49 & 0.06 & $0.93(0.88-0.96)$ & 33 & 0.12 & $0.76(0.56-0.87)$ \\
\hline
\end{tabular}

Abbreviations: BA, brachial artery; CI, confidence interval; CR, coefficient of repeatability; DPA, dorsalis pedis artery ICC, intraclass correlation coefficient; PTA, posterior tibial artery. 


\section{References}

[1] Fowkes FG. Epidemiological research on peripheral vascular disease. J Clin Epidemiol 2001;54:863-8.

[2] Shinozaki T, Hasegawa T, Yano E. Ankle-arm index as an indicator of atherosclerosis: its application as a screening method. J Clin Epidemiol 1998;51:1263-9.

[3] Stoffers HE, Kester AD, Kaiser V, Rinkens PE, Kitslaar PJ, Knottnerus JA. The diagnostic value of the measurement of the ankle-brachial systolic pressure index in primary health care. J Clin Epidemiol 1996;49:1401-5.

[4] Greenland P, Abrams J, Aurigemma GP, Bond MG, Clark LT, Criqui MH, Crouse JR 3rd, Friedman L, Fuster V, Herrington DM, Kuller LH, Ridker PM, Roberts WC, Stanford W, Stone N, Swan HJ, Taubert KA, Wexler L; Writing Group III. Prevention Conference V: Beyond secondary prevention: identifying the high-risk patient for primary prevention: noninvasive tests of atherosclerotic burden. Circulation 2000;101:E16-22.

[5] Criqui MH, Langer RD, Fronek A, Feigelson HS, Klauber MR, McCann TJ, Browner D. Mortality over a period of 10 years in patients with peripheral arterial disease. N Engl J Med 1992;326: $381-6$.

[6] Leng GC, Fowkes FG, Lee AJ, Dunbar J, Housley E, Ruckley CV. Use of ankle brachial pressure index to predict cardiovascular events and death: a cohort study. BMJ 1996;313:1440-4.

[7] Hooi JD, Stoffers HE, van Kester AD, van Ree J, Knottnerus JA. Peripheral arterial occlusive disease: prognostic value of signs, symptoms, and the ankle-brachial pressure index. Med Decis Making 2002;22:99-107.

[8] Vogt MT, Cauley JA, Newman AB, Kuller LH, Hulley SB. Decreased ankle/arm blood pressure index and mortality in elderly women. JAMA 1993;270:465-9.

[9] Newman AB, Shemanski L, Manolio TA, Cushman M, Mittelmark M, Polak JF, Powe NR, Siscovick D. Relationship between coronary artery calcification and other measures of subclinical cardiovascular disease in older adults. Arterioscler Thromb Vasc Biol 2002;22:1674-9.

[10] Boker LK, van Noord PA, van der Schouw YT, Koot NV, de Mesquita HB, Riboli E, Grobbee DE, Peeters PH; European Prospective Investigation into Cancer and Nutrition. Prospect-EPIC Utrecht: study design and characteristics of the cohort population. Eur J Epidemiol 2001;17:1047-53.

[11] Bland JM, Altman DG. Statistical methods for assessing agreement between two methods of clinical measurement. Lancet 1986;1(8476): 307-10.

[12] Bailey RH, Bauer JH. A review of common errors in the indirect measurement of blood pressure: sphygmomanometry. Arch Intern Med 1993;153:2741-8.

[13] Bennett S. Blood pressure measurement error: its effect on cross-sectional and trend analyses. J Clin Epidemiol 1994;47: 293-301.

[14] Andersen UO, Henriksen JH, Jensen G. Sources of measurement variation in blood pressure in large-scale epidemiological surveys with follow-up. Blood Press 2002;11:357-65.
[15] Elliott WJ. Circadian variation in blood pressure: implications for the elderly patient. Am J Hypertens 1999;12:43S-9S.

[16] Mancia G, Grassi G. Mechanisms and clinical implications of blood pressure variability. J Cardiovasc Pharmacol 2000;35:S15-9.

[17] Fagan TC, Conrad KA, Mayshar PV, Mackie MJ, Hagaman RM. Single versus triplicate measurements of blood pressure and heart rate. Hypertension 1988;11:282-4.

[18] Fowkes FG, Housley E, Macintyre CC, Prescott RJ, Ruckley CV. Variability of ankle and brachial systolic pressures in the measurement of atherosclerotic peripheral arterial disease. J Epidemiol Community Health 1988;42:128-33.

[19] McDermott MM, Criqui MH, Liu K, Guralnik JM, Greenland P, Martin GJ, Pearce W. Lower ankle/brachial index, as calculated by averaging the dorsalis pedis and posterior tibial arterial pressures, and association with leg functioning in peripheral arterial disease. $\mathbf{J}$ Vasc Surg 2000;32:1164-71.

[20] Aboyans V, Lacroix P, Lebourdon A, Preux PM, Ferrieres J, Laskar M. The intra- and interobserver variability of ankle-arm blood pressure index according to its mode of calculation. J Clin Epidemiol 2003;56:215-20.

[21] Aboyans V, Lacroix P, Preux PM, Vergnenegre A, Ferrieres J, Laskar M. Variability of ankle-arm index in general population according to its mode of calculation. Int Angiol 2002;21:237-43.

[22] Sacks D, Bakal CW, Beatty PT, Becker GJ, Cardella JF, Raabe RD, Wiener HM, Lewis CA. Position statement on the use of the ankle-brachial index in the evaluation of patients with peripheral vascular disease: a consensus statement developed by the standards division of the society of cardiovascular \& interventional radiology. J Vasc Interv Radiol 2002;13:353.

[23] Jeelani NU, Braithwaite BD, Tomlin C, MacSweeney ST. Variation of method for measurement of brachial artery pressure significantly affects ankle-brachial pressure index values. Eur J Vasc Endovasc Surg 2000;20:25-8.

[24] Kaiser V, Kester AD, Stoffers HE, Kitslaar PJ, Knottnerus JA. The influence of experience on the reproducibility of the ankle-brachial systolic pressure ratio in peripheral arterial occlusive disease. Eur J Vasc Endovasc Surg 1999;18:25-9.

[25] Mätzke S, Franckena M, Alback A, Railo M, Lepantalo M. Ankle brachial index measurements in critical leg ischaemia: the influence of experience on reproducibility. Scand J Surg 2003;92:144-7.

[26] Orme S, Ralph SG, Birchall A, Lawson-Matthew P, McLean K, Channer KS. The normal range for inter-arm differences in blood pressure. Age Ageing 1999;28:537-42.

[27] Lane D, Beevers M, Barnes N, Bourne J, John A, Malins S, Beevers DG. Inter-arm differences in blood pressure: when are they clinically significant? J Hypertens 2002;20:1089-95.

[28] Singer AJ, Hollander JE. Blood pressure: assessment of interarm differences. Arch Intern Med 1996;156:2005-8.

[29] Perloff D, Grim C, Flack J, Frohlich ED, Hill M, McDonald M, Morgenstern BZ. Human blood pressure determination by sphygmomanometry. Circulation 1993;88:2460-70.

[30] The fifth report of the Joint National Committee on Detection, Evaluation, and Treatment of High Blood Pressure (JNC V). Arch Intern Med 1993;153:154-83. 